\title{
The Analysis for the Two-stage Model on Scale-free Networks
}

\author{
Maoxing Liu, Yunli Zhang \\ Department of Mathematics North University of China Taiyuan, P.R. China, 030051
}

\begin{abstract}
In this paper, we will study a two-stage model by complex networks. The dynamic behaviors of the model on a heterogenous scale-free (SF) network are considered, where the absence of the threshold on the SF network is demonstrated, and the stability of the disease-free equilibrium is obtained.
\end{abstract}

Index Terms: Complex network; Two-stage model; Epidemic; Threshold

(C) 2011 Published by MECS Publisher. Selection and/or peer review under responsibility of the Research Association of Modern Education and Computer Science.

\section{Introduction}

Mathematical models have been applied to the study of infectious diseases since more than a century ago. The last four decades have witnessed a burst of interest in quantitatively understanding the transmission dynamics of a large number of diseases [1]. One of the key aims of epidemiological mathematical models, and certainly the most relevant in terms of policy making, is the assessment of the effectiveness of control strategies to curb disease spreading. For many infectious diseases, the most widespread prevention measure is mass vaccination. However, if for a given disease vaccines are not known, or vaccination is not effective, other control measures have to be adopted.

Compartmental model is often used to describe the spread of infectious diseases, such as HIV, rabies, SARS, TB. In 1927, Kermack and McKendrick suggested an SIR model, which well indicated the number of infected population during the plague [2]. The contact process was introduced by Harris [3]. In a contact process, individuals in the population have two life stages, young and adult, only adults can give birth and each new offspring is young. Transition from young to adult occurs at constant rate, and individuals die at rates that depend on their life stage. Krone [6] gives a very nice analysis of this process by the construction of a multi-type dual process. Models of this class have proven particularly useful for the study of Chlamydia and gonorrhea [4], [5].

We consider a population formed by $\mathrm{N}$ agents, situated at the nodes of a network. Individuals are modeled as nodes, and possible contacts between individuals are linked by edges. It has been shown that there is an

* Corresponding author.

E-mail address: liumaoxing@126.com, zhangyunli5365@sina.com 
epidemic threshold on the homogeneous Watts-Strogatz (WS) small-world network model, while the epidemic model on SF networks has no epidemic threshold; infections can be proliferated, whatever small infection rate they have. This result disproves the threshold theory in epidemiology. Recently, there are some related research works on complex networks, for example, with infective medium [14], even with two species or pathogens spreading [15], [16].

These studies have extended and detailed the epidemic model on complex networks. It has shown that networks of two-stage contact process are scale-free [6], [7], [8], and [17] i.e. the distribution of the number of contacts over all individuals in the network has a long upper tail which exhibits power-law behavior. To study such a spreading characteristic, this paper extends the standard model to qualitatively understand and describe the mechanism of epidemics spreading based on biological means. It is shown that the main features and theoretical results obtained here are different from those in general SIS models [7], [8], [9].

The state-transition rules of the two-stage contact process have been put forward in Ref. [17]. It differs from the SIRS model in that the transition rate from 0 to 1 is proportional to the density of the neighbors with state 2 (not 1 ) of a state- 0 vertex. This model corresponds to dynamics with two life stages. The states can be interpreted as 0: vacant, 1: occupied by young individuals, and 2: occupied by adults. Only adults are reproductive and generate offspring in neighboring vacant sites at a birth rate equal to $\lambda$ In other words, a birth event occurs at a vacant site at a rate proportional to $\lambda$ and the number of neighboring adults. Youngs (state 1) spend random time of mean $\gamma$ before becoming adult (state 2). They are also subject to random death events at a rate of $\delta$. Adults die at a rate of 1 , which gives normalization of the entire model. Alternatively, we can interpret the three states as 0: vacant, 1: partially occupied, and 2: fully occupied colonies. Then, only fully occupied colonies are potent enough to colonize vacant lands. Based on these state-transition rules, we will establish and consider this model on scale-free networks.

The organization of this paper is as follows: Section 2 derives the mean-field epidemic spreading model of SF network, especially Barabasi-Albert(BA) model with connectivity $p(k) \square k^{-3}$ is obtained, and the existence of the steady-state solution is analyzed. Section 3 discusses the stability of the disease-free equilibrium. Finally, a brief discussion is given in Section 4.

\section{The model}

To derive mean field dynamics for populations with heterogeneous contact rates, let us denote by $p_{k}$ the probability that a vertex has degree $k$. Obviously, $\sum_{k=1}^{N} p_{k}=1$.

In this paper, there are two types of nodes in a network, one is composed of $N$ first-stage individuals and the other is composed of $N$ second-stage individuals. All nodes can exist only in one of the two discrete states, healthy or infected. And the disease transmission is described as in the paper [7], [8]:

At each time step, each susceptible (healthy) node is infected with rate $v$ if it is connected to one or more infected nodes.

At the same time, infected nodes are cured and become again susceptible with rate ${ }^{\tau}$ defining an effective spreading rate

$\lambda=v / \tau$. Without lack of generality, we can set $\tau=1$. All nodes in this network run stochastically through the healthy-infected-healthy cycles. So in this paper, we are interested in studying the following dynamical model 


$$
\begin{aligned}
& \dot{\rho}_{1, k}=\lambda\left(1-\rho_{1, k}-\rho_{2, k}\right) k \Theta_{2}-(\delta+\gamma) \rho_{1, k}, \\
& \dot{\rho}_{2, k}=\gamma \rho_{1, k}-\rho_{2, k} .
\end{aligned}
$$

we note that the effective birth rate (the first term in the first equation (1a) is proportional to $k \Theta_{2}$, which is the average number of state- 2 vertices in the neighborhood of a degree- $k$ vertex. When we choose an arbitrary edge, the probability that a specific vertex is connected to this edge is proportional to its degree $k$ [7], [8], [10], [11], [13], where $\rho_{1, k}\left(\rho_{2, k}\right)$ denotes the density of infected first-stage and second-stage individuals with connectivity $\mathrm{k}$ in the networks, the edge-conditioned probability $0 \cdot £ 2(\mathrm{t}) \cdot 1$ describes a link pointing to an infected individual [7], [8], which satisfies:

$$
\Theta_{2}(t)=\frac{\sum_{k} k p_{k} \rho_{2, k}(t)}{\langle k\rangle} .
$$

where $\langle k\rangle=\sum_{k} k p_{k}, p_{k}$ is the connectivity distribution of the vertex individuals.

In the steady state

$$
\dot{\rho}_{1, k}=0, \dot{\rho}_{2, k}=0
$$

one has

$$
\begin{aligned}
& \lambda\left(1-\rho_{1, k}-\rho_{2, k}\right) k \Theta_{2}-(\delta+\gamma) \rho_{1, k}=0, \\
& \gamma \rho_{1, k}-\rho_{2, k}=0 .
\end{aligned}
$$

it is easy to see that model has a disease-free equilibrium: $E_{0}=(0, \cdots, 0)$. As far as the possibility of spreading is concerned, the following lemma holds:

Theorem 2.1. If and only if

$\lambda>\frac{(\delta+\gamma)\langle k\rangle}{\gamma\left\langle k^{2}\right\rangle}$,

then there exists a unique endemic equilibrium $E^{*}=\left(x_{1}^{*}, \cdots, x_{N}^{*}, y_{1}^{*}, \cdots, y_{N}^{*}\right)$.

proof: From (4), we can get

$$
\begin{aligned}
\rho_{1, k} & =\frac{\lambda k \Theta_{2}}{\lambda(1+\gamma) k \Theta_{2}+\delta+\gamma}, \\
\rho_{2, k} & =\frac{\lambda \gamma k \Theta_{2}}{\lambda(1+\gamma) k \Theta_{2}+\delta+\gamma} .
\end{aligned}
$$

And substituting them into (2), we get

$$
\Theta_{2}=\frac{1}{\langle k\rangle} \sum_{k} \frac{\lambda \gamma k^{2} \Theta_{2} p_{k}}{\lambda(1+\gamma) k \Theta_{2}+\delta+\gamma} \square f\left(\Theta_{2}\right) \text {. }
$$

For Eq.(6) is satisfied when $\Theta_{2}=0$, corresponding to the disease-free state. When $0<\Theta_{2}<1$ state 2 survives, Eq.(6) implies $\rho_{1, k}>0, \rho_{2, k}>0$ in this situation. Accordingly, $\Theta_{2}>0$ is equivalent to the $\{0,1,2\}$ 
phase. It is easy to verify that $f\left(\Theta_{2}\right)$ is a convex and increase function, and $\left.f\left(\Theta_{2}\right)\right|_{\Theta_{2}=1}<1$, so if there is another solution $0<\Theta_{2}<1$, it must satisfy $\left.\frac{d f\left(\Theta_{2}\right)}{d \Theta_{2}}\right|_{\Theta_{2}=0}>1$,

that is

$$
\lambda>\frac{(\delta+\gamma)\langle k\rangle}{\gamma\left\langle k^{2}\right\rangle}
$$

where $\left\langle k^{2}\right\rangle=\sum_{k} k^{2} p_{k}$. Through the unique endemic equilibrium $E^{*}$ is determined. This agrees with the results for the percolation, the contact process, and the SIR model.

Remark 2.1.

(1) Let

$$
R=\frac{\lambda \gamma\left\langle k^{2}\right\rangle}{(\delta+\gamma)\langle k\rangle}
$$

In mathematical biology, $R$ is called the basic productive number, which determines whether the disease is transmittable or not.

(2) If we consider $p_{k}=C k^{-v} \delta_{k, k_{c}}\left(k_{c} \geq 2\right)$. The network is homogenous, $\langle k\rangle=k_{c}$, so

$$
R=\frac{\lambda \gamma k_{c}}{\delta+\gamma}
$$

When $R<1$, the disease-free equilibrium is local asymptotically stable, while $R>1$, there is an endemic equilibrium. Moreover, $R$ is an increasing function of $\lambda$ and $k_{c}$. In order to make $R<1$, we need decrease $\lambda$ and $k_{c}$. (3) By using a continuous $k$ approximation that allows a practical substitution of series with integrals [18], the full connectivity distribution can be obtained as $p_{k}=2 m^{2} / k^{3}$, where $m$ is the minimum number of connections at each individual node, and

$$
\langle k\rangle=\int_{m}^{\infty} k p_{k} d k=2 m .
$$

Furthermore, here

$$
\left\langle k^{2}\right\rangle \square 2 m^{2} \ln \left(k_{c} / m\right)
$$

Substituting them into yields

$$
R=\frac{\lambda \gamma m \ln \left(k_{c} / m\right)}{\delta+\gamma} .
$$

If we consider the maximum connectivity $k_{c}$ to $N$ by $k_{c} m \sqrt{N}$, so

$R=\frac{\lambda \gamma m \ln (N)}{2(\delta+\gamma)}$.

\section{The stability of the disease-free equilibrium (dfe)}


In this section we consider the stability of the disease-free equilibrium. First, Jacobin matrix at an equilibrium is

$$
J=\left(\begin{array}{ll}
A & B \\
C & D
\end{array}\right),
$$

where

$$
A=\left(\begin{array}{ccc}
-\lambda \Theta_{2}-(\delta+\gamma) & \cdots & 0 \\
\vdots & \ddots & \vdots \\
0 & \cdots & -N \lambda \Theta_{2}-(\delta+\gamma)
\end{array}\right)
$$

$B=B_{1}+B_{2}$, where

$$
\begin{gathered}
B_{1}=\left(\begin{array}{ccc}
-\lambda \Theta_{2} & \cdots & 0 \\
\vdots & \ddots & \vdots \\
0 & \cdots & -N \lambda \Theta_{2}
\end{array}\right), \\
B_{2}=\frac{\lambda}{\langle k\rangle}\left(\begin{array}{ccc}
p_{1} h_{1} & \cdots & N p_{N} h_{N} \\
\vdots & \ddots & \vdots \\
N p_{1} h_{N} & \cdots & N^{2} p_{N} h_{N}
\end{array}\right),
\end{gathered}
$$

where $h_{i}=1-\rho_{1, i}-\rho_{2, i},(i=1, \cdots, N)$.

So we can easily get the Jacobin matrix at disease-free

$$
\begin{aligned}
& C=\gamma\left(\begin{array}{ccc}
1 & \cdots & 0 \\
\vdots & \ddots & \vdots \\
0 & \cdots & 1
\end{array}\right), \\
& D=\left(\begin{array}{ccc}
-1 & \cdots & 0 \\
\vdots & \ddots & \vdots \\
0 & \cdots & -1
\end{array}\right) .
\end{aligned}
$$
equilibrium is

$$
J^{\prime}=\left(\begin{array}{ll}
A^{\prime} & B^{\prime} \\
C^{\prime} & D^{\prime}
\end{array}\right),
$$

where

$$
A^{\prime}=\left(\begin{array}{ccc}
-(\delta+\gamma) & \cdots & 0 \\
\vdots & \ddots & \vdots \\
0 & \cdots & -(\delta+\gamma)
\end{array}\right)
$$




$$
\begin{gathered}
B^{\prime}=\frac{\lambda}{\langle k\rangle}\left(\begin{array}{ccc}
p_{1} & \cdots & N p_{N} \\
\vdots & \ddots & \vdots \\
N p_{1} & \cdots & N^{2} p_{N}
\end{array}\right), \\
C^{\prime}=\gamma\left(\begin{array}{ccc}
1 & \cdots & 0 \\
\vdots & \ddots & \vdots \\
0 & \cdots & 1
\end{array}\right), \\
D^{\prime}=\left(\begin{array}{ccc}
-1 & \cdots & 0 \\
\vdots & \ddots & \vdots \\
0 & \cdots & -1
\end{array}\right) .
\end{gathered}
$$

The matrix $J^{\prime}$ has $2 N-1$ eigenvalues equal to $; 1: \mu_{i}=-1(i=1, \cdots 2 N-1)$, the $2 N$ th is

$$
\mu_{2 N}=-1+\frac{\lambda \gamma\left\langle k^{2}\right\rangle}{(\delta+\gamma)\langle k\rangle} .
$$

Having established these premises, we may immediately demonstrate the following theorem:

Theorem 3.1. If $R \leq 1$ then the disease-free equilibrium of (1) is asymptotically stable in the set $[0,1]^{2 N}$, otherwise there exists a unique endemic equilibrium $E^{*}=\left(x_{1}^{*}, \cdots, x_{N}^{*}, y_{1}^{*}, \cdots, y_{N}^{*}\right)$.

\section{Remark 3.1.}

The biological consequence of this result is that if the epidemic threshold $R$ is not exceeded the disease will disappear. On the contrary if $R>1$, then there is an endemic solution which is reached independently of the initial state of the disease. In other words, however complex the system may be and whatever the initial state of the first-stage and the second-stage individuals are, whether the disease will eradicate or not only depend on the basic reproduction number.

\section{Conclusion}

In this paper, a new model for two-stage contact process on complex networks has been proposed and simulated. Different from the classical epidemic model, in the new model infection between two-stage contact processes is taken into account. Moreover, spreading thresholds of the two-stage contact process model are determined, and the stability of the disease-free equilibrium is given, but as to the stability of the endemic equilibrium, which can show the global behavior of the solutions of the model, we have not mentioned in this paper. These and some other related issues will be further studied in the future.

\section{Acknowledgment}

The authors would like to thank the referee for his/her valuable comments which have led to an improvement of the presentation of this paper. 


\section{References}

[1] R. M. Anderson, R. M. May, Infectious Diseases of Humans, Oxford University Press, Oxford, UK (1991).

[2] Kermack, W. O. and A. G. McKendrick. 1927. A Contribution to the Mathematical. Theory of Epidemics. Proc. Roy. Soc. A. 115, 700-721.

[3] T. Harris, Contact interactions on a lattice, Ann. Probab., 2 (1974) 969-988.

[4] H. W. Hethcote, J. A. Yorke, Gonorrhoea: transmission dynamics and control in: Lecture Notes in Biomathematics, vol. 56. Springer, New York, 1984.

[5] M. Kretzschmar, Y.T.H.P. Van Duynhoven, A. J. Severijnen, Modeling prevention strategies for gonorrhoea and chlamydia using stochastic network simulations. Am. J. Epidemiol., 144 (1997) 306-317.

[6] S. M. Krone, The two-stage contact process, The Annals of Applied Probability, 9 (2) 1999 331-351.

[7] R. Pastor-Satorras, A. Vespignani, Epidemic spreading in scale-free networks, Phys. Rev. Lett., 86 (2001a) 3200-3203.

[8] R. Pastor-Satorras, A. Vespignani, Epidemic dynamics and endemic states in complex networks, Phys. Rev. E, 63 (2001b) 066117.

[9] R. Pastor-Satorras, A. Vespignani, Immunization of complex networks, Phys. Rev. E, 65 (2002) 036104.

[10] D. S. Callaway, M. E. J. Newman, S. H. Strogatz, D. J. Watts, Network robustness and fragility: percolation on random graphs, Phys. Rev. Lett.,85 (2000) 5468-5471.

[11] R. Cohen, K. Erez, D. Ben-Avraham, S. Havlin, Resilience of the Internet to random breakdowns, Phys. Rev. Lett., 85 (2000) 4626-4628.

[12] R. Cohen, S. Havlin, D. Ben-Avraham, , Phys. Rev. Lett., 91 (2003) 247901.

[13] M. E. J. Newman, Spread of epidemic disease on networks, Phys. Rev. E, 66 (2002) 016128.

[14] H. Shi, Z. Duan, G. Chen, An SIS model with infective medium on complex networks, Physica A 387 (2008) 2133-2144.

[15] Y. Y. Ahn, H. Jeong, N. Masuda, J. D. Noh, Epidemic dynamics of two species of interacting particles on scale-free networks, Phys. Rev. E, 74 (2006) 066113.

[16] M. E. J. Newman, Threshold Effects for Two Pathogens Spreading on a Network, Phys. Rev. Lett., 95 (2005) 108701.

[17] N.K. Masuda, N. Konno, Multi-state epidemic processes on complex networks, Journal of Theoretical Biology, 243 (2006) 64-75.

[18] A. -L. Barabasi, R. Albert, Emergence of scaling in random networks, Science 286 (1999) 509-512.

[19] W. P. Guo, X. Li, X. F. Wang, Epidemics and immunization on Euclidean distance preferred small-world networks, Physica A 380 (2007) 684-690.

[20] X. L, X. F. Wang, Controlling the spreading in small-world evolving networks: stability, oscillation, and topology, IEEE Trans. Automat.Control 51 (3) (2006) 534-540.

[21] X. L, X. F. Wang, On the stability of epidemic spreading in small-world networks: how prompt the recovery should be?, Int. J. Syst. Sci. 38 (5) (2007) 400-407. 